\title{
Antibiotic susceptibility of ica-positive and ica-negative MRSA in different phases of biofilm growth
}

\author{
Shivani Chopra, Kusum Harjai and Sanjay Chhibber
}

Methicillin-resistant Staphylococcus aureus (MRSA) has emerged as a clinically relevant pathogen because of its resistance to antibiotics and its ability to form potent biofilm. Both ica-negative as well as ica-positive MRSA strains are known to produce biofilm. In the present study, these strains were grown in biofilm mode and susceptibility of these to antibiotics was assessed. Our study suggests that antibiotic susceptibility of MRSA biofilm depends on the biochemical composition of its matrix. The biofilm matrix of ica-positive MRSA was mainly composed of poly-intercellular adhesion (PIA), whereas eDNA was a major constituent of ica-negative MRSA. The results showed that MRSA in planktonic growth was susceptible to clindamycin, vancomycin and minocycline. However, the MIC and MBC of vancomycin for the mature biofilm of ica-negative MRSA was 16 and $32 \mu \mathrm{g} \mathrm{ml}^{-1}$, respectively. On the contrary, the MIC and MBC of vancomycin for ica-positive MRSA was $>1024 \mu \mathrm{gml}-1$. The effect of vancomycin and minocycline on young and old biofilms was also determined. Vancomycin was quite effective in eradicating the young biofilm formed by ica-negative MRSA; however, it was completely ineffective on the biofilm of ica-positive MRSA. Minocycline at its highest clinical achievable concentration was found to be quite effective in eradicating the young biofilm formed by both the strains. The enzyme-linked immunosorbent assay (ELISA) results and dot blot assay suggest that the presence of ica locus influenced PIA production, which probably contributed towards the failure of vancomycin in eradicating the biofilm formed by ica-positive strain. However, none of the antibiotics used in this study was effective in eradicating the mature biofilms.

The Journal of Antibiotics (2015) 68, 15-22; doi:10.1038/ja.2014.96; published online 30 July 2014

\section{INTRODUCTION}

Methicillin-resistant $S$. aureus (MRSA) strains pose a major threat to patients because of their resistance towards $\beta$-lactam antibiotics such as oxacillin. MRSA has spread worldwide both in health-care and community settings. ${ }^{1}$ Presently, the prevalence of methicillin resistance among $S$. aureus isolates in intensive care units in the United States is $60 \%,{ }^{2}$ and more than 90000 invasive infections due to MRSA occur in the United States. ${ }^{3}$ Moreover in recent years, the prevalence of MRSA in India has increased from 12\% in 1992 to $80.83 \%$ in 2002.4

Over the years, vancomycin has been considered as a gold standard treatment for MRSA infections. It is generally considered as the first-line option for the treatment of bacteremia, endocarditis, osteomyelitis, septic arthritis and meningitis, followed by antibiotics such as clindamycin, doxycycline, minocycline and linezolid. ${ }^{5}$ However, to compound this problem, MRSA is also known to form potent biofilm. ${ }^{1}$

Earlier, only poly-intercellular adhesion (PIA) molecules endcoded by ica-operon were known to be responsible for the biofilm-forming ability of $S$. aureus. However, now it is known that an ica ADBCindependent biofilm phenotype, which instead depends upon the fibronectin-binding proteins (FnBPA and FnBPB) and major autoysin (Atl), also exists. ${ }^{6-10}$ Hence, in addition to resistance towards antibiotics, the biofilm formed on the implanted prosthetic biomaterials is a major risk factor in health-care-associated infections. Moreover, during the course of infection, the implant becomes coated with host-derived extracellular matrix proteins, ${ }^{11}$ resulting in increased antibiotic resistance of biofilm cells that is up to 1000 -fold greater than the free-living planktonic bacterial cells. ${ }^{12-14}$ The existence of microbial cells in stationary phase and permeability barrier imposed by aggregative biopolymers that hold the biofilm together contribute towards resistance to antimicrobials. ${ }^{13,14}$

Till date, no regimen has been established to treat chronic biofilm infections that differ markedly from planktonic cells grown in the same environment. According to the Clinical and Laboratory Standards Institute (CLSI) methods, the killing dose determined for antibiotics is prepared only for planktonic cells, as a result of which they have no effect on bacteria grown within biofilm. ${ }^{15-17}$ The minimum biofilm eradication concentration, which targets complete bacterial killing, would be more meaningful than the biofilm MIC to prevent chronic phase of biofilm infections, which are characterized by less active dispersal. ${ }^{12,15}$ The minimum concentration required for 
biofilm eradication is impractical to achieve when conventional antibiotics are used in vitro ${ }^{18,19}$ but the biofilm MBC, which targets 99.9\% killing of biofilm-grown bacteria, appears to be achievable.

To the best of our knowledge, no report is yet available in which a comparative study has been conducted to evaluate the significance of ica-locus in the context of antibiotic susceptibility of different phases of planktonic as well as biofilm growth. In the present study, the biofilm formation has been characterized and quantified in presence and absence of the ica-locus. In addition, antibiotic susceptibility of various phases of biofilm growth of MRSA has been studied (planktonic cells at the mid-log phase, planktonic cells at the stationary phase and mature biofilms) at their serum achievable concentration.

\section{MATERIALS AND METHODS}

\section{Bacterial strains}

MRSA ATCC 33591 and ATCC 43300 procured from American type Culture Collection Centre, Manassas, VA, USA were used in the present study. All the strains were preserved in semi-solid agar stabs at $4{ }^{\circ} \mathrm{C}$ and in brain heart infusion broth with $15 \%$ glycerol at $-70^{\circ} \mathrm{C}$ until further use. All antimicrobial susceptibility experiments were performed in soyabean casein digest medium. Growth media and antibiotics were purchased from HiMedia Laboratories (Mumbai, India). Nutrient agar was used as the culture medium for MRSA and bacterial dilutions were made in sterile $0.85 \%$, normal saline (NS).

\section{Antibiotics}

Vancomycin hydrochloride, clindamycin and minocycline (HiMedia Laboratories) representing members of glycopeptides, lincosamide and broad-range tetracycline were used in the present study.

\section{Biofilm development}

MRSA biofilm was grown in 96-well microtiter plate according to the modified method of Bedi et al. ${ }^{20}$ Briefly, wells of microtiter plate were inoculated with $50 \mu \mathrm{l}$ of soyabean casein digest medium and $50 \mu \mathrm{l}$ of bacterial culture $\left(\mathrm{OD}_{600}=0.3\right)$ equivalent to $10^{8} \mathrm{CFU} \mathrm{ml}^{-1}$ of bacteria and incubated at $37^{\circ} \mathrm{C}$ overnight. In each test, control wells containing sterile broth were included as plate sterility control. After incubation, planktonic cells in the fluid were removed and wells were washed thoroughly three times with NS. The biofilm matrix was then scraped with a sterile pipette tip, suspended in NS and vortexed for $3 \mathrm{~min}$. Microbial load of biofilm was enumerated by viable cell counting.

For the establishment of 7-day-old biofilm, spent medium was replaced with fresh sterile soyabean casein digest medium in rest of the wells and plate was reincubated at $37^{\circ} \mathrm{C}$ overnight. Media in each well was replaced every $24 \mathrm{~h}$ until the seventh day of the experiment. Every day, wells were stained with $0.1 \%$ crystal violet for $10 \mathrm{~min}$. Following the staining step, the colorant was discarded and the wells were rinsed three times with NS to remove excess stain. Then, the dye incorporated in the biofilm or present in the control wells was solubilized in absolute ethanol. The OD of the stained biofilm and control wells was read at $595 \mathrm{~nm}$ using enzyme-linked immunosorbent assay reader (BIORAD iMark Microplate Reader, Bio-Rad Laboratories Inc, Hercules, CA, USA). Microbial load of the biofilm was also enumerated daily by viable cell count method till 7 days.

\section{Slime production}

The slime production was assessed by the method of Freeman et al. ${ }^{21}$ Briefly, the plates composed of brain heart infusion agar and Congo red $0.8 \mathrm{gl}^{-1}$ were inoculated and incubated aerobically for $24 \mathrm{~h}$ at $37^{\circ} \mathrm{C}$. A positive result was indicated on the basis of colony morphology. A black colony with a dry crystalline consistency was considered positive. Non-slime producers usually remained pink, although occasional darkening at the center of the colony was observed and this was labeled as bull's eye appearance.

\section{Assessment of biochemical nature of biofilm}

The composition of biofilm matrix was assessed by the method of Fischer et al..$^{22}$ Briefly, wells of microtiter plate were inoculated with $50 \mu$ of soyabean casein digest medium and $50 \mu \mathrm{l}$ of bacterial culture $\left(\mathrm{OD}_{600}=0.3\right)$ equivalent to $10^{8} \mathrm{CFU} \mathrm{ml}{ }^{-1}$ of bacteria and incubated at $37^{\circ} \mathrm{C}$ overnight. In each test, $0.1 \mathrm{U}$ DNase I and Proteinase K $\left(0.2 \mathrm{mg} \mathrm{ml}^{-1}\right.$ in $100 \mathrm{~mm}$ Tris $\mathrm{NaCl}$, pH 7.5) were added and plates were incubated for $24 \mathrm{~h}$ at $37^{\circ} \mathrm{C}$ without agitation. After incubation, planktonic cells in the fluid were removed and wells were washed thoroughly three times with NS. After drying the biofilm, the test and control wells stained with CV as described above. The OD of the stained biofilm and control wells was read at $595 \mathrm{~nm}$ using enzyme-linked immunosorbent assay reader (BIORAD iMark Microplate Reader, Bio-Rad Laboratories Inc). In addition, the amount of eDNA and protein content was assessed by measuring $\mathrm{OD}_{260}$ and $\mathrm{OD}_{280}$ using UV Nanodrop (ThermoFischer Scientific Ltd., Waltham, MA, USA).

\section{Quantification of eDNA and proteins in biofilm}

The amount of eDNA was quantified by following the method of Vilain et al. ${ }^{23}$ Briefly, biofilm was established as described above. Each day the wells were washed three times with NS. The biofilm cells were scraped and centrifuged at 10000 r.p.m. for $10 \mathrm{~min}$ at $4{ }^{\circ} \mathrm{C}$. The supernatant was filtered by using $0.45-\mu \mathrm{m}$ syringe filter (Millipore Ireland Ltd., Tullagreen, Carrigtwohill, County Cork) to remove all the bacterial cells. The amount of eDNA present in the supernatant was assessed by measuring O.D $D_{260}$ using UV Nanodrop 1000 (ThermoFischer Scientific Ltd.).

\section{PIA quantification using dot blot and ELISA}

PIA was extracted from biofilm according to the method of Jefferson and Cerca. ${ }^{24}$ Briefly, the biofilm was grown on $35-\mathrm{mm}$ dishes, scraped off and suspended in phosphate-buffered saline (PBS). The $\mathrm{OD}_{600}$ of the suspension was adjusted to 1.5 , centrifuged at $9296 \mathrm{~g}$ for $5 \mathrm{~min}$ and the pellet was suspended in $50 \mathrm{ml} 0.5 \mathrm{M}$ EDTA ( $\mathrm{pH} 8.0$ ). The samples were incubated, twice at $100{ }^{\circ} \mathrm{C}$ for $5 \mathrm{~min}$ with incubation on ice for $5 \mathrm{~min}$ in between and then centrifuged at $9296 \mathrm{~g}$ for $5 \mathrm{~min}$. The supernatant was treated with proteinase $\mathrm{K}$ $\left(2 \mathrm{mg} \mathrm{ml}^{-1}\right)$ for $1 \mathrm{~h}$ at $60^{\circ} \mathrm{C}$ and then incubated at $80^{\circ} \mathrm{C}$ for $30 \mathrm{~min}$ to inactivate proteinase $\mathrm{K}$. The samples were stored at $-20^{\circ} \mathrm{C}$ till further use.

For dot blot, twofold dilutions of PIA extract were prepared in Tris-buffered saline (TBS) and $5 \mu \mathrm{l}$ of each suspension was spotted on nitrocellulose membrane. The membrane was air-dried, moistened with TBS and fixed in 50 -ml blocking solution comprising $1 \%(\mathrm{w} / \mathrm{v})$ bovine serum albumin (BSA) in TBST (TBS plus $0.05 \%$ Tween 20 ) for $1 \mathrm{~h}$. Fifty milliliters of WGA-horseradish peroxidase conjugate (WGA-HRP, $130 \mathrm{ng} \mathrm{ml}^{-1}$ ) plus $1 \%$ BSA in TBST were added and the membrane was incubated for $30 \mathrm{~min}$. The membrane was then washed three times for $5 \mathrm{~min}$ each in TBST, incubated in $50 \mathrm{ml}$ substrate (0.5 mg diaminobenzidine per ml plus $1 \mu \mathrm{H}_{2} \mathrm{O}_{2}$ per $\mathrm{ml}$ in TBS) for $15 \mathrm{~min}$ and rinsed in distilled water. The experiment was performed in duplicate.

PIA in biofilm was quantitated by enzyme-linked immunosorbent assay (ELISA) according to the method of Cramton et al. ${ }^{25}$ Overnight cultures of both the strains were adjusted to an $\mathrm{OD}_{600}$ of 0.3 , and $200 \mu \mathrm{l}$ of this culture was incubated per well in flat-bottomed, 96-well polystyrene microtiter plates for $24 \mathrm{~h}$ at $37^{\circ} \mathrm{C}$. The resulting biofilm was washed twice with PBS and fixed with $200 \mu \mathrm{l}$ of $95 \%$ ethanol for $15 \mathrm{~min}$. An aliquot of $100 \mu \mathrm{l}$ blocking solution $(1 \%, w / v$, BSA in PBST (PBS plus $0.05 \%$ Tween 20$)$ ) was added per well and the microtiter plate incubated for $1 \mathrm{~h}$ at $37^{\circ} \mathrm{C}$. The blocking solution was then removed, $100 \mu \mathrm{l}$ WGA-HRP $\left(75 \mathrm{ng} \mathrm{ml}^{-1}\right)$ in $1 \%$ BSA in PBST was added per well and the microtiter plate incubated for $30 \mathrm{~min}$ at $37^{\circ} \mathrm{C}$. The wells were washed three times for $5 \mathrm{~min}$ each in PBST. Substrate $(100 \mu \mathrm{l} 0.5 \mathrm{mg}$ orthophenylenediamine per ml plus $5 \mu \mathrm{H}_{2} \mathrm{O}_{2}$ per ml in citrate buffer (pH 5.0)) was then added, the microtitre plate was incubated for $5 \mathrm{~min}$ at $37^{\circ} \mathrm{C}$ and the reaction was stopped by adding $50 \mu \mathrm{l}$ of $12.5 \% \mathrm{H}_{2} \mathrm{SO}_{4}$ per well. The $\mathrm{A}_{490}$ was measured. Experiments were performed in duplicate.

\section{Antibiotic susceptibility testing}

MIC and MBC of planktonic cells at the mid-log phase. The MIC of the planktonic cells was determined in accordance with the Clinical and Laboratory Standards Institute (formerly NCCLS) guidelines. ${ }^{26}$ Antibiotics 
a

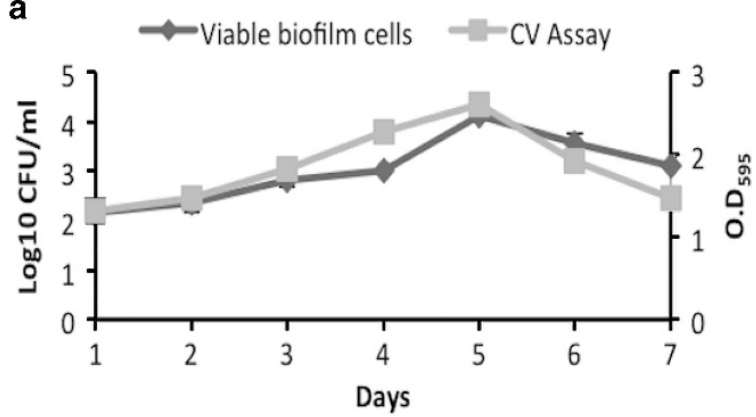

b

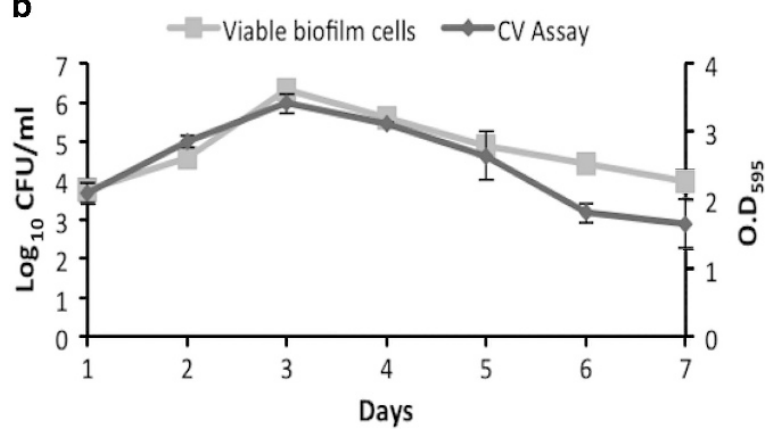

Figure $1(\mathbf{a}, \mathbf{b})$ Biofilm-forming ability of ica-negative and ica-positive MRSA over a period of 7 days as determined by crystal voilet staining $\left(\mathrm{OD}_{595}\right)$ and viable biofilm cell quantification. $\mathrm{CV}$, coefficient of variation.

were used at a concentration ranging from 1024 to $0.062 \mathrm{mg}^{-1}$. The highest dilution of the antibiotic showing visible inhibition of bacterial growth after overnight incubation at $37^{\circ} \mathrm{C}$ was taken as MIC of the drug.

The MBC of the antibiotics against planktonic bacteria at mid-log phase was determined by plating the contents of the tubes containing MIC and higher concentration of antibiotic on nutrient agar and incubated at $37^{\circ} \mathrm{C}$ for $24 \mathrm{~h}$. Before plating, the bacteria with or without antibiotic treatment were pelleted with centrifugation at 10000 r.p.m. for $10 \mathrm{~min}$, washed twice with NS and finally suspended in $1 \mathrm{ml}$ NS. The resulting cell suspension was serially diluted, and viable count was enumerated by drop plating.

MIC and MBC of biofilm cells. The method of Ceri et al. ${ }^{15}$ was used to determine MIC of biofilm-grown bacteria. Biofilm established in 96-well microplate was exposed to $100 \mu \mathrm{l}$ of twofold serial dilution of antibiotics ranging from 1024 to $0.062 \mathrm{mg} \mathrm{ml}^{-1}$ in Tryptose Soy Broth. The microplate was then incubated at $37^{\circ} \mathrm{C}$ for $24 \mathrm{~h}$. After overnight challenge, the supernatant from each well was carefully transferred to the wells in a new 96-well microplate without disturbing the biofilm, and the turbidity of the contents was visually assessed. The biofilm MIC was defined as the lowest concentration of antibiotic at which no visible growth was observed. When exposed to antibiotics at or above this concentration, the biofilm-shed bacteria showed susceptibility to antibiotic.

MBC of the biofilm bacteria was also assessed on the basis of reduction in viable counts after overnight exposure to antibiotics. The viable bacteria remaining in biofilm in wells containing MIC or higher concentrations of antibiotic were counted by plating on nutrient agar and incubating at $37^{\circ} \mathrm{C}$ for $24 \mathrm{~h}$. MBC of biofilm was calculated by dividing the predetermined viable count of untreated biofilm by viable count obtained after antibiotic exposure.

MBC of planktonic cells at the stationary phase. MIC of antibiotic at stationary phase could not be tested by standard procedure because of high cell density of bacteria in the overnight culture. The MBC of antibiotics against planktonic bacteria at the stationary phase was determined following the Clinical and Laboratory Standards Institute guidelines, ${ }^{27}$ except that stationary phase culture replaced log-phase culture. The MBCs for planktonic cells at the

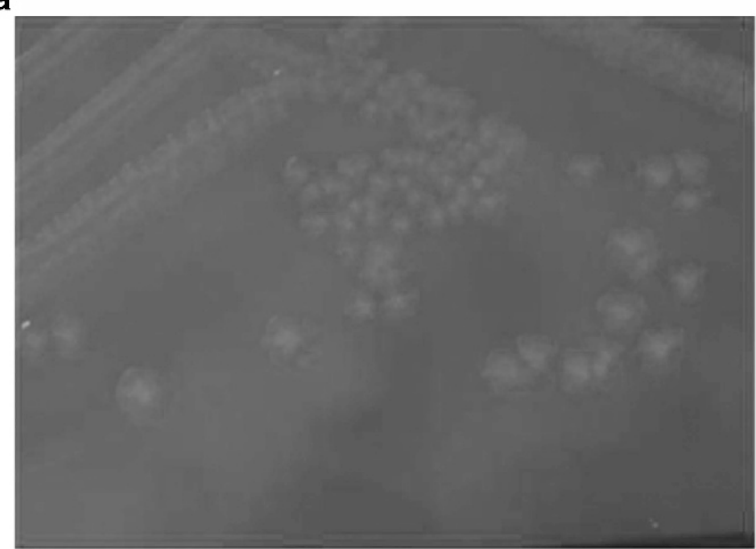

b

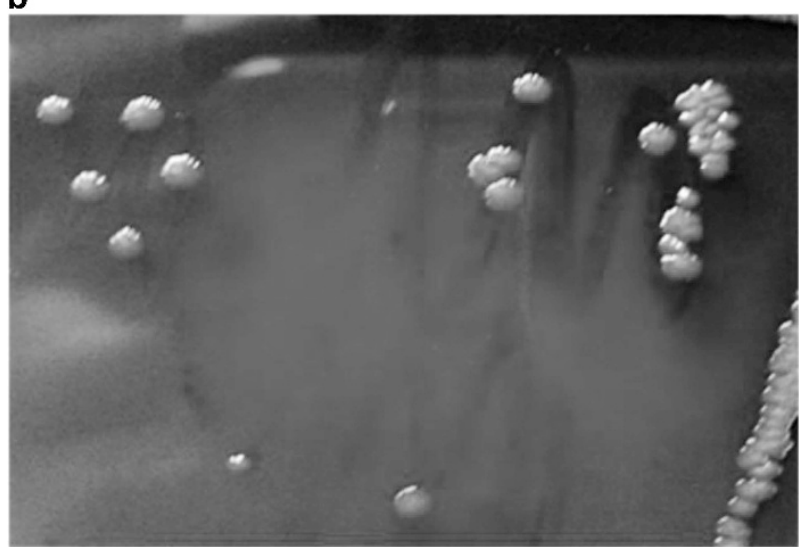

Figure $2(\mathbf{a}, \mathbf{b})$ Colony morphology of ica-negative and ica-positive MRSA on Congo red Agar. A full color version of this figure is available at The Journal of Antibiotics journal online.

stationary phase and for biofilm was defined as the minimum concentration of antibiotics required to reduce bacterial numbers by at least 3 logs.

Effect of antibiotics on preformed biofilm. As the maximum clinically achievable concentration of vancomycin and minocycline is 60 and $4 \mu \mathrm{g} \mathrm{ml}^{-1}$, respectively ${ }^{28}$ (intravenously), the concentration used in the susceptibility assay was 60 and $150 \mu \mathrm{g} \mathrm{ml}^{-1}$ for vancomycin and $4 \mu \mathrm{g} \mathrm{ml}^{-1}$ for minocycline. Initially, the control and untreated wells were incubated at $37^{\circ} \mathrm{C}$ overnight. For each subsequent day antibiotic was added to the well for that day for which it was to be tested. (For example on day 1, antibiotic was added to well untreated for 1 day, on day 2 antibiotic was added to well untreated for 2 days and so on for 7 days.) After the overnight treatment, the viable count was enumerated using drop-plating method. ${ }^{29}$ Experiments were performed in duplicate.

\section{Statistical analysis}

All the experiments were performed in duplicate. The mean and s.d. were calculated from the results. The bacterial count was converted to $\log _{10}$. The effect of different treatments on biofilm eradication was evaluated by two-way analysis of variance and Student's $t$-test, and $P<0.01$ was considered significant.

\section{RESULTS}

The results of biofilm formed by ica-positive and ica-negative MRSA are shown in Figures 1a and b. In case of ica-positive MRSA, the biofilm formation peaked on the third day of incubation, whereas in ica-negative MRSA maximum the biofilm formation was seen on the fifth day of incubation. Following this, a decrease in biofilm 
formation was observed as bacterial counts declined with time. The results of semiquantitative estimation were also confirmed by quantification of viable biofilm cells.

\section{Comparison of slime production and biochemical composition}

The slime production by both strains under study was assessed by culturing them on CRA plates. As shown in Figures $2 a$ and b, ica-negative MRSA gave pink to red colonies, whereas ica-positive MRSA showed black colonies with dry crystalline consistency. Further incubation up to $72 \mathrm{~h}$ to check delayed slime production by ica-negative MRSA showed no significant change in color or darkening of the center. On treating the ica-negative MRSA biofilm with DNase and ProteinaseK and subsequently staining it with CV, it was observed that biofilm-forming ability of ica-negative MRSA was reduced in comparison with control. As shown in Figure $3 \mathrm{a}$, the biofilm-forming ability of ica-negative MRSA plunged on days 1, 2 and 3. The biofilm formation was negligible in comparison with control on days 5 (peak day), 6 and 7. However, in case of ica-positive MRSA (Figure $3 \mathrm{~b}$ ), treatment with DNase and Proteinase K led to a decrease in $\mathrm{OD}_{595}$ from 3.41 to 1.36 on day 3 (peak day). Similarly, $\mathrm{OD}_{595}$ decreased from 1.81 and 1.65 to 1.05 and 1.05 on days 6 and 7 , respectively.
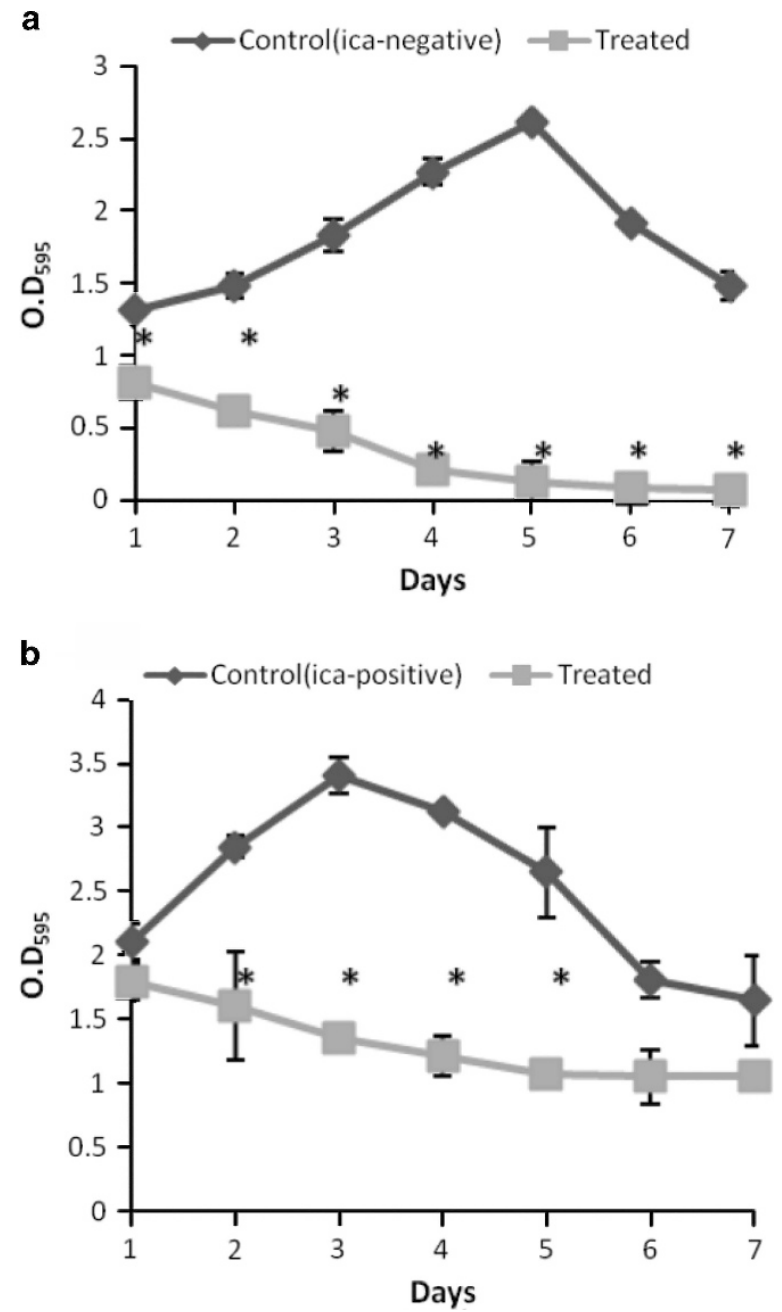

Figure $3(\mathbf{a}, \mathbf{b})$ Susceptibility pattern of ica-negative and ica-positive MRSA biofilms to DNase and ProteinaseK. Experiment was performed in duplicate. Error barrs indicate s.d. ${ }^{*} P<0.01$.

\section{Quantification of eDNA in biofilm}

The quantity of eDNA released by ica-negative MRSA biofilm was assessed over the period of 7 days. An increase in the amount of eDNA release was seen over time and the observed increase was $92.2 \pm 0.08 \mathrm{ng}^{-1} \mathrm{l}^{-1}$ on day 1 to $144.1 \pm 0.05 \mathrm{ng} \mu \mathrm{l}^{-1}$ on day 5 (peak day). The amount of eDNA was $132.72 \pm 0.3$ and $130.41 \pm$ $0.11 \mathrm{ng} \mu \mathrm{l}^{-1}$, respectively, on days 6 and 7 , showing no further change in eDNA release.

\section{Estimation of PIA using dot blot and ELISA}

The PIA production by ica-positive MRSA was assessed both using dot blot and ELISA. In dot blot assay, the amount of PIA was quantified on days 1, 3, 5 and 7. As shown in Figure 4, the PIA signal was detected upto 1:64 on day 7. In addition, PIA was quantified using ELISA on days 1, 3, 5 and 7. PIA production was found to increase with time (day 1: $1.83 \pm 0.04 ; \mathrm{A}_{490} \pm$ s.d.) and the maximum amount $(2.33 \pm 0.09)$ was seen on the third day (that is, peak day), after which no significant $(P<0.01)$ decrease (day 5: $2.29 \pm 0.08)$ was observed in PIA amount (day 7: $2.28 \pm 0.03$ ).

\section{Antibiotic susceptibility of MRSA biofilm at different stages of formation}

Differences in MIC and MBC of planktonic cultures and biofilm cells of ica-negative MRSA. The MIC and MBC of clindamycin for biofilm cells was significantly higher than log planktonic cells (that is, $>1024 \mu \mathrm{g} \mathrm{ml}^{-1}$ ), which was more than the upper detection limit as shown in Table 1. On the contrary, no significant difference in the MIC and MBC of vancomycin and minocycline for biofilm cells was observed when we compared them with log planktonic cells. The MBC of clindamycin for biofilm cells and stationary planktonic cells was more than the upper detection limit. In addition, no significant decrease in MIC and MBC of biofilm cells for vancomycin and minocycline was observed in comparison with stationary planktonic cells.

Differences in MIC and MBC of planktonic cultures and biofilm cells of ica-positive MRSA. The MBC of clindamycin and vancomycin for biofilm cells was more than the highest concentration tested in comparison with log planktonic cells. On the contrary, the MIC of

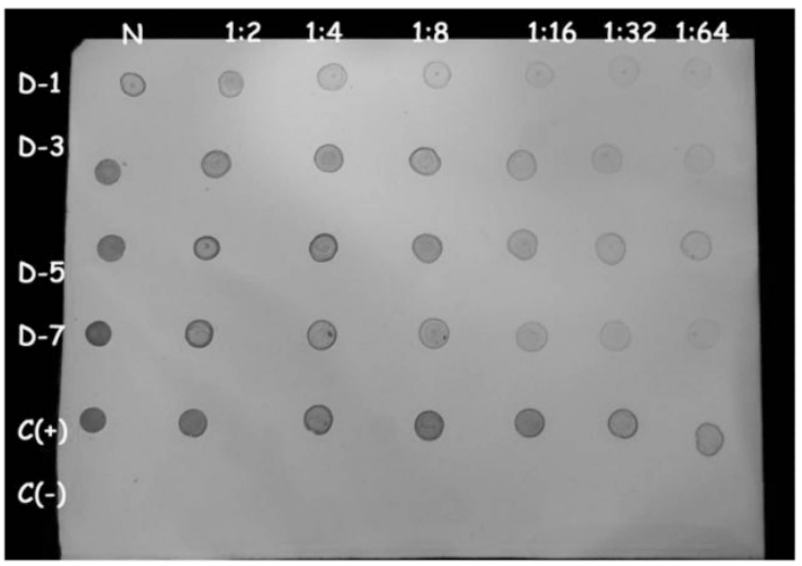

Figure 4 Semi-quantitative estimation of PIA in the biofilm formed by ica-positive MRSA on day $1,3,5$ and 7 by dot blot. PIA was detected using WGA-HRP. S. aureus ATCC 12598 and S. epidermidis ATCC 12228 were used as positive $(+)$ and negative $(-)$ controls, respectively. N, Neat (undiluted) extract. A full color version of this figure is available at The Journal of Antibiotics journal online. 
Table 1 Antibiotic susceptibility of three antibiotics against MRSA biofilms in different phases of biofilm formation

\begin{tabular}{|c|c|c|c|c|c|c|c|}
\hline \multirow[b]{2}{*}{ Mode of growth } & \multirow[b]{2}{*}{ Initial bacterial density $\left(\mathrm{CFU} \mathrm{ml}^{-1}\right)$} & \multicolumn{2}{|c|}{ Clindamycin $\left(\mu g \mathrm{ml}^{-1}\right)$} & \multicolumn{2}{|c|}{ Vancomycin $\left(\mu g \mathrm{~m} /^{-1}\right)$} & \multicolumn{2}{|c|}{ Minocycline $\left(\mu g m l^{-1}\right)$} \\
\hline & & MIC & $M B C$ & MIC & $M B C$ & MIC & $M B C$ \\
\hline Log planktonic & $1.2 \times 10^{5}$ & 64 & 128 & 8 & 16 & 0.25 & 1 \\
\hline Stationary planktonic & $1.1 \times 10^{7}$ & - & $>1024$ & - & 16 & - & 4 \\
\hline Mature biofilm & $1.3 \times 10^{7}$ & $>1024$ & $>1024$ & 16 & 32 & 0.5 & 2 \\
\hline Log planktonic & $3.4 \times 10^{5}$ & 128 & 256 & 8 & 64 & 0.5 & 2 \\
\hline Stationary planktonic & $3.5 \times 10^{8}$ & - & 512 & - & 256 & - & 8 \\
\hline Mature biofilm & $3.2 \times 10^{8}$ & $>1024$ & $>1024$ & 512 & $>1024$ & 8 & 16 \\
\hline
\end{tabular}

Abbreviation: MRSA, Methicillin-resistant Staphylococcus aureus.
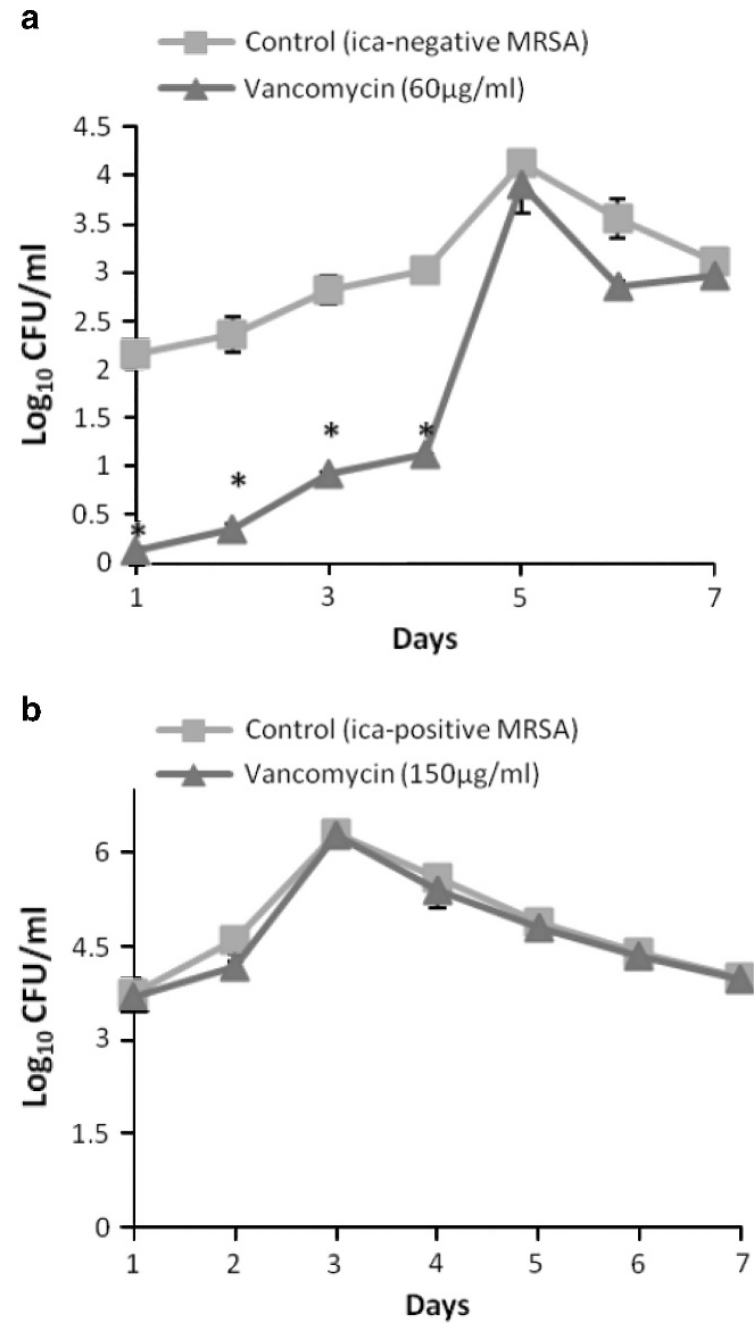

Figure 5 (a, b) Susceptibility pattern of ica-negative and ica-positive MRSA to vancomycin on different days of incubation. Experiment was performed in duplicates. Error bars indicate s.d. ${ }^{*} P<0.0 .1$.

vancomycin and minocycline for biofilm cells was 64 and 16 times higher, respectively, when compared with log planktonic cells (Table 1). However, no significant decrease in the MBC of vancomycin and minocycline for biofilm cells and stationary planktonic cells was observed.
Differences in MIC and MBC of both types of biofilms. As shown in Table 1, the MIC of vancomycin and minocycline for ica-positive MRSA biofilm was 32 and 16 times, respectively, higher than ica-negative MRSA biofilm. However, clindamycin was ineffective in eradicating both types of biofilms as the MIC and MBC were found to be higher than the upper detection limit. In addition, the $\mathrm{MBC}$ of vancomycin was $>1024 \mu \mathrm{g} \mathrm{ml}^{-1}$ for ica-positive MRSA biofilm.

\section{Effect of antibiotics on preformed biofilm}

Susceptibility of preformed MRSA biofilms to vancomycin. The effect of vancomycin was studied on both the ica-positive as well as ica-negative preformed biofilms. The results showed that a concentration of $60 \mu \mathrm{g} \mathrm{ml}^{-1}$, which is also its highest clinical achievable concentration, was found to be quite effective in eradicating the biofilm formed by ica-negative MRSA (Figure 5a). A significant decrease of 2.02 logs and $2.00 \operatorname{logs}(P<0.01)$ was observed on day 1 and day 2 , respectively. A uniform decrease was found on next consecutive days. However, a nonsignificant decrease of $0.71 \operatorname{logs}$ and $0.15 \operatorname{logs}(P>0.01)$ was observed in the bacterial count of older biofilms (that is, peak day onwards). On the contrary, as shown in Figure 5b, vancomycin was completely ineffective in eradicating the biofilm produced by icapositive MRSA biofilm even at a concentration higher than its serum achievable concentration. No significant reduction in biofilm bacterial count $(P>0.01)$ was observed from day 1 to day 7 .

Susceptibility of MRSA biofilm to minocycline. The results of susceptibility of MRSA biofilm to minocycline are shown in Figures $6 \mathrm{a}$ and $\mathrm{b}$. Minocycline was found to be quite effective on young biofilm formed by ica-negative MRSA at a concentration of $4 \mu \mathrm{g} \mathrm{ml}^{-1}$, which is also its highest clinical achievable concentration. A significant gradual decrease in $\log$ values was detected from day 1 to day 4 , and corresponding values on each day were found to be as 2.3, 2.26, 2.10 and 2.00 logs, respectively. A significant decrease of 1.90 logs was also observed on the peak day. However, no significant reduction in bacterial count was observed in older biofilm. Similarly, minocycline was able to eradicate the biofilm formed by ica-positive MRSA. On day 1, a significant decrease of 2.00 logs was observed. A reduction in bacterial count upto 2.03 logs was also observed on day3 (that is, the peak day). However, minocycline was ineffective when used on older biofilms of both the MRSA strains.

\section{DISCUSSION}

Majority of infections are attributed to biofilm mode of growth. ${ }^{30}$ However, antibiotics fail to eradicate these cells as concentration of 
a
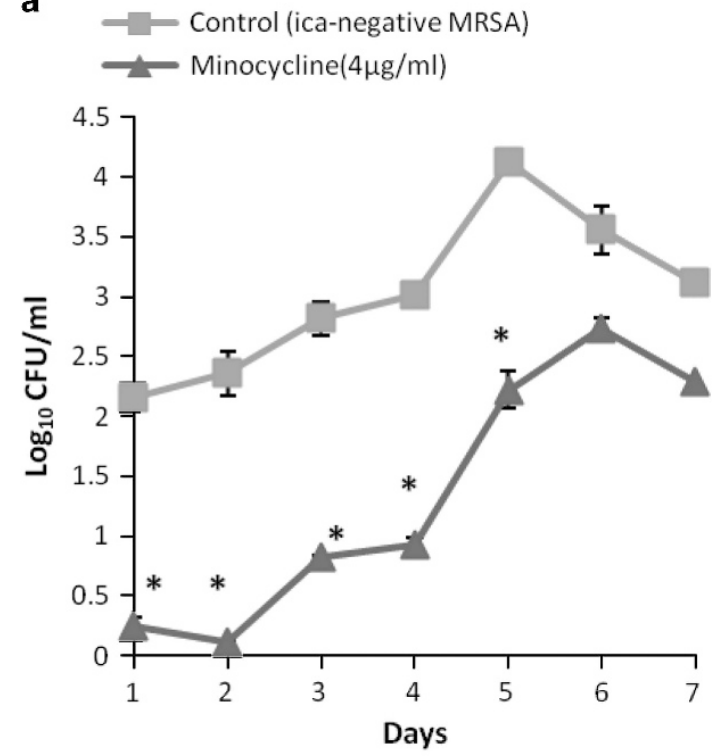

b



Figure 6 (a, b) Antibiotic susceptibility pattern of ica-negative MRSA and ica-positive MRSA biofilm to minocycline. Experiment was performed in duplicate. Error bars indicate s.d. ${ }^{*} P<0.01$.

antibiotic required to eradicate bacteria in biofilm is much higher than MIC of planktonic cells. In this study, an attempt was made to describe a system that may help in elucidating the effectiveness of different classes of antibiotics in the eradication of biofilm produced by MRSA strains with or without ica-locus.

Two standard MRSA strains (ATCC MRSA 43300 possessing ica-locus ${ }^{31}$ and ATCC MRSA 33591 lacking ica-locus ${ }^{32}$ ) were compared on the basis of their biofilm-forming ability, its biochemical/morphological characterization and antibiotic susceptibility. The different phases of growth relevant to biofilm formation were included. MRSA strain lacking ica-locus in comparison with ica-positive MRSA took longer time in establishing itself in biofilm mode. These results suggest that absence ica-locus most probably is a contributing factor in delaying the biofilm formation. Similar results have been proposed previously showing that ica-negative S. epidermidis was weak biofilm producer and took comparatively longer time in establishing its biofilm mode than ica-positive strains. $^{33}$ To further confirm this finding, both the MRSA strains were assessed for their ability to produce slime. Slime not only helps the organism in their attachment to different surfaces but also hinders the penetration of antibiotic by acting as a chemical barrier to them. The difference in colony morphology on CRA agar confirmed the ability of ica-positive MRSA to produce comparatively more slime than the ica-negative MRSA.

The biochemical composition of biofilm produced by both the strains was significantly altered when treated with proteinase $\mathrm{K}$ and DNase, showing that biofilm matrix in both the cases was rich in protein. However, the biofilm-forming ability of ica-negative MRSA was significantly less in presence of DNase vis a vis ica-positive-treated biofilm. Hence, the eDNA content in the biofilm of ica-negative MRSA was evaluated. The results showed that eDNA was the major constituent of ica-negative MRSA biofilm. In recent study Fisher et al. $^{22}$ have also demonstrated eDNA to be the major component of biofilm matrix in ica-independent $S$. aureus biofilm. On the other hand, PIA was found to be the major component of ica-dependent $S$. aureus biofilm. The further confirmation was made by estimating the amount of PIA and eDNA. In ica-positive MRSA biofilm, an increase in PIA with successive increase in biofilm age was observed. The amount of PIA produced by ica-positive MRSA was maximum on the peak day of biofilm formation (that is, third day). Similar increase in the content of eDNA was observed in ica-negative MRSA biofilm showing maximum amount of eDNA on the peak day of biofilm formation (that is, fifth day).

The planktonic cultures in the mid-log phase, stationary phase and mature biofilm were assessed for their susceptibility to different classes of antibiotics. The rationale was to compare the MIC and MBC of antibiotics for planktonic and biofilm growth of both the strains and compare the antibiotic susceptibility of two types of biofilm. Our study showed that both the strains in their log planktonic phase were susceptible to clindamycin, vancomycin and minocycline. However, no significant difference was observed in the $\mathrm{MIC}$ and $\mathrm{MBC}$ of stationary planktonic and mature biofilm. This is consistent with the findings of earlier workers (Spoering and Lewis, ${ }^{34}$ Qu et al. ${ }^{35}$ and Singla et al. ${ }^{36}$ ) who also showed that stationary-phase culture possessed showed similar or higher resistance than the biofilm-grown bacteria.

On comparing the MIC and MBC of two types of biofilms, some interesting results were seen. Significant difference was observed in the $\mathrm{MIC}$ and $\mathrm{MBC}$ of vancomycin for stationary planktonic and mature biofilm of ica-positive and ica-negative MRSA. The reason for this difference probably lies in the composition of the biofilm matrix of both the biofilms. As mentioned earlier, the biofilm matrix of icapositive MRSA had a dense structure, mainly composed of PIA and vancomycin being a density-dependent antibiotic was not able to penetrate into it effectively. As observed in this study, as well as reported earlier, the ica-negative S. epidermidis forms weak biofilm in the absence of ica-locus. Thus, lack of PIA results in less thicker biofilm, which in turn allows more effective penetration of antibiotic. ${ }^{37}$ On the other hand, antibiotic belonging to the tetracycline group was found to be quite effective in eradicating both ica-positive as well as ica-negative MRSA biofilms. In the earlier study, Cerca et al. ${ }^{38}$ also showed tetracyclines to be more effective than cell wall synthesis inhibiting antibiotics because antibiotics that target RNA and protein syntheses have similar activities on planktonic and biofilm cells. Hence, a significant decrease in the young biofilm of ica-positive and ica-negative MRSA was observed in presence of minoycline. In addition, in ica-negative MRSA biofilms Atl (major 
autolysin) and FnBPA and FnBPB proteins have also been found to be major factors. ${ }^{6-10}$ According to previous findings of Yamada et al. ${ }^{39}$ and Ledala et al. ${ }^{40}$ tetracyclines suppress the localization of Atl and this in turn lead to impaired autolysin activity. This major autolysin (Atl) not only helps in initial attachment of biofilm to various surfaces but also helps in the lysis of bacteria to release eDNA in the biofilm matrix. ${ }^{41}$

Biofilm age is also a decisive factor in determining the outcome of a therapeutic regimen. ${ }^{36,42}$ Older biofilms are known to require higher antibiotic concentrations for their eradication than planktonic cells as well as younger biofilm. ${ }^{43}$ Our study showed that vancomycin at its highest serum achievable concentration was quite effective in eradicating the young biofilm formed by ica-negative MRSA. On the contrary, the antibiotic failed to eradicate the biofilm formed by ica-positive MRSA. Even when used at a higher concentration (that is, $150 \mu \mathrm{g} \mathrm{ml}^{-1}$ ), it failed to give significant decrease in the number of biofilm cells. On the other hand, using minocycline at its serum achievable concentration both the biofilms were effectively eradicated. Minocycline significantly decreased the biofilm cell count in young biofilm of ica-positive MRSA (that is, upto 2 days) and ica-negative (that is, upto 4 days) MRSA. Both the antibiotics, however, were ineffective in eradicating the older biofilm. This might be because of the inaccessibility of antibiotic to bacteria as an increase in extracellular matrix was observed in older biofilm. ${ }^{44,45}$

Our study confirms that biofilm kinetics, morphological characteristics and antibiotic sucsceptibility of MRSA biofilm are dependent upon the biochemical composition of the biofilm. The production of PIA in significant amount altered the antibiotic susceptibility of the isolates confirming that it was dependent upon the presence of ica-locus. The cell wall-inhibiting antibiotic such as vancomycin was not effective against ica-positive biofilm cells but the same could eradicate the biofilm produced by ica-negative MRSA. On the other hand, tertracylines (minocycline) appeared to be most effective to treat young biofilm of both the MRSA strains. The results of this study also suggest that early initiation of treatment of bacteria with antibiotics in the biofilm mode is essential because as the biofilm grows older, there is increased production of extracellular polysaccharide and metabolically less active cells. These changes make the older biofilm resistant to antibiotics. However, the data generated in this study are based on the observations made on the standard strains of MRSA. It is suggested that future studies should concentrate on wild-type isolates from clinical sources so as to have a more meaningful data.

1 O'Gara, J. P. ica and beyond: biofilm mechanisms and regulation in Staphylococcus epidermidis and Staphylococcus aureus. FEMS Microbiol. Lett. 270, 179-188 (2007).

2 National Nosocomial Infections Surveillance System. National Nosocomial Infections Surveillance (NNIS) System Report, data summary from January 1992 through June 2004, issued October 2004. Am. J. Infect. Control 32, 470 (2004).

3 Klevens, R. M. et al. Invasive methicillin-resistant Staphylococcus aureus infections in the United States. JAMA 298, 1763 (2007).

4 Verma, S., Joshi, S., Chitnis, V., Hemwani, N. \& Chitnis, D. Growing problem of methicillin resistant staphylococci - Indian scenario. Indian J. Med. Sci. 54, 535-540 (2000).

5 Liu, C. et al. Clinical practice guidelines by the Infectious Diseases Society of America for the treatment of methicillin resistant Staphylococcus aureus infections in adults and children. Clin. Infect. Dis. 52, e18-e55 (2011).

6 Beenken, K. E. et al. Global gene expression in Staphylococcus aureus biofilms. J. Bacteriol. 186, 4665-4684 (2004).

7 Fitzpatrick, F., Humphreys, H. \& O'Gara, J. P. Evidence for icaADBC independent biofilm development mechanism in methicillin-resistant Staphylococcus aureus clinical isolates. J. Clin. Microbiol. 43, 1973-1976 (2005).

8 O'Neill, E. et al. Association between methicillin susceptibility and biofilm regulation in Staphylococcus aureus isolates from device-related infections. J. Clin. Microbiol. 45, 1379-1388 (2007).
9 Hennig, S., Nyunt, W. S. \& Ziebuhr, W. Spontaneous switch to PIA independent biofilm formation in an ica-positive Staphylococcus epidermidis isolate. Int. J. Med. Microbiol. 297, 117-122 (2007).

100 'Neill, E. et al. A novel Staphylococcus aureus biofilm phenotype mediated by the fibronectin-binding proteins, FnBPA and FnBPB. J. Bacteriol. 190, 3835-3850 (2008).

11 Herrmann, M. et al. Fibronectin, fibrinogen and laminin act as mediators of adherence of clinical staphylococcal isolates to foreign material. J. Infect. Dis. 158, 693-701 (1988).

12 Ceri, H., Olson, M. E., Morck, D. W., Read, R. R. \& Buret, A. G. The Calgary Biofilm Device: measurement of antimicrobial sensitivity of bacterial biofilms. J. Clin. Microbiol. 37, 1771-1776 (1999).

13 Gilbert, P., McBain, A. \& Rickard, A. H. in: Multiple Drug Resistant Bacteria (eds Amábile-Cuevas, C. F.) 81-107 (Horizon Scientific Press, London, UK, 2003).

14 Wentland, E. J., Stewart, P. S., Huang, C. T. \& McFeters, G. A. Spatial variations in growth rate within Klebsiella pneumoniae colonies and biofilm. Biotechnol. Prog. 12, 316-321 (1996).

15 Ceri, H. et al. The MBEC Assay System: multiple equivalent biofilms for antibiotic and biocide susceptibility testing. Methods Enzymol. 337, 377-385 (2001).

16 Moskowitz, S. M., Foster, J. M., Emerson, J. \& Burns, J. L. Clinically feasible biofilm susceptibility assay for isolates of Pseudomonas aeruginosa from patients with cystic fibrosis. J. Clin. Microbiol. 42, 1915-1922 (2004).

17 Pettit, R. K. et al. Microplate Alamar blue assay for Staphylococcus epidermidis biofilm susceptibility testing. Antimicrob. Agents Chemother. 49, 2612-2617 (2005).

18 Cheung, G. Y. \& Otto, M. Understanding the significance of Staphylococcus epidermidis bacteremia in babies and children. Curr. Opin. Infect. Dis. 23, 208-216 (2010).

19 Qu, Y., Istivan, T. S., Daley, A. J., Rouch, D. A. \& Deighton, M. A. Comparison of various antimicrobial agents as catheter lock solutions: preference for ethanol in eradication of coagulase-negative staphylococcal biofilms. J. Med. Microbiol. 58 , 442-450 (2009).

20 Bedi, M. S., Verma, V. \& Chhibber, S. Amoxicillin and specific bacteriophage can be used together for eradication of biofilm of Klebsiella pneumoniae B5055. World J. Microbiol. Biotechnol. 25, 1145-1151 (2009).

21 Freeman, D. J., Falkiner, F. R. \& Keane, C. T. New method for detecting slime production by coagulase negative staphylococci. J. Clin. Pathol. 42, 872-874 (1989).

22 Fischer, A. et al. GdpS contributes to Staphylococcus aureus biofilm formation by regulation of eDNA release. Int. J. Med. Microbiol. 304, 284-299 (2014).

23 Vilain, S., Pretorius, J. M., Theron, J. \& Broz̈el, V. S. DNA as an Adhesin: Bacillus cereus requires extracellular DNA to form biofilms. Appl. Environ. Microbiol. 75, 2861-2868 (2009).

24 Jefferson, K. K. \& Cerca, N. Bacterial-bacterial cell interactions in biofilms: detection of polysaccharide intercellular adhesins by blotting and confocal microscopy. Methods Mol. Biol. 341, 119-126 (2006).

25 Cramton, S. E., Ulrich, M., Gotz, F. \& Doring, G. Anaerobic conditions induce expression of polysaccharide intercellular adhesion in Staphylococcus aureus and Staphylococcus epidermidis. Infect. Immun. 69, 4079-4085 (2001).

26 Clinical and Laboratory Standards Institute. Performance Standards for Antimicrobial Susceptibility Testing: Sixteenth Informational Supplement M100-S16 (CLSI, Wayne, PA, USA, 2006)

27 Clinical and Laboratory Standards Institute. Methods for Dilution Antimicrobial Susceptibility Tests for Bacteria that Grow Aerobically-. 7th edn, Approved standard M7-A7 (CLSI, Wayne, PA, USA, 2004).

28 Yao, J. D. C. \& Moellering, R. C. Jr. in: Manual of Clinical Microbiology Antibacterial Agents (eds Murray, P. R., Baron, E. J., Pfaller, M. A., Jorgensen, J. H. \& Yolken, R. H.) 1039-1073 (American Society for Microbiology, Washington, DC, USA, 2003).

29 Miles, A. A., Misra, S. S. \& Irwin, J. O. The estimation of the bactericidal power of the blood. J. Hyg. (Lond) 38, 732-749 (1938).

$30 \mathrm{NIH}$, N. H. Lung and Blood Institute Reasearch on Microbial Biofilms (2002) [http:// www.nhlbi.nih.gov]

$31 \mathrm{Tan}, \mathrm{H}$. et al. The use of quaternised chitosan-loaded PMMA to inhibit biofilm formation and downregulate the virulence-associated gene expression of antibioticresistant staphylococcus. Biomaterials 33, 365-377 (2012).

32 Iorio, N. L. M. Methicillin-resistant Staphylococcus epidermidis carrying biofilm formation genes: detection of clinical isolates by multiplex PCR. Int. Microbiol. 14 (Suppl 1), 13-17 (2011).

33 Dice, B. et al. Biofilm formation by ica-positive and ica-negative strains of Staphylococcus epidermidis in vitro. Biofouling 25(Suppl 4), 367-375 (2009).

34 Spoering, A. L. \& Lewis, K. Biofilms and planktonic cells of Pseudomonas aeruginosa have similar resistance to killing by antimicrobials. J. Bacteriol. 183, 6746-6751 (2001).

35 Qu, Y., Daley, A. J., Istivan, T. S., Garland, S. M. \& Deighton, M. A. Antibiotic susceptibility of coagulase-negative staphylococci isolated from very low birth weight babies: comprehensive comparisons of bacteria at different stages of biofilm formation. Ann. Clin. Microbiol. Antimicrob. 9, 16 (2010).

36 Singla, S., Harjai, K. \& Chhibber, S. Susceptibility of different phases of biofilm of Klebsiella pneumoniae to three different antibiotics. J. Antibiot. 66, 61-66 (2013).

37 Mack, D. et al. The intercellular adhesion involved in biofilm accumulation of Staphylococcus epidermidis is a linear beta-1,6 linked glucosaminoglycan: purification and structural analysis. J. Bacteriol. 178, 175-183 (1996).

38 Cerca, N. Comparative assessment of antibiotic susceptibility of coagulase negative staphylococci in biofilm versus planktonic culture as assessed by bacterial enumeration or rapid XTT colorimetry. J. Antimicrob. Chemother. 56, 331-336 (2005). 
39 Yamada, S., Sugai, M., Komatsuzawa, H. \& Matsumato, A. Suppressed localisation of a major autolysin on Staphylococcus aureus treated with tetracycline. J. Electron Microsc. 50, 359-364 (2001).

40 Ledala, N., Wilkinson, B. J. \& Jayaswal, R. K. Effects of oxacillin and tetracycline on autolysis, autolysin processing and at/ transcription in Staphylococcus aureus. Int. J. Antimicrob. Agents 27, 518-524 (2006)

41 Arciola, C. R., Campoccia, D., Speziale, P., Costerton, J. W. \& Montanaro, L. Biofilm formation in Staphylococcus implants infections. A review of molecular mechanisms and implications for biofilm-resistant materials. Biomaterials $\mathbf{3 3}$ 5967-5982 (2012).
42 Sedlacek, M. J. \& Walker, C. Antibiotic resistance in an in vitro subgingival biofilm model. Oral Microbiol. Immunol. 22, 333-339 (2007).

43 Ito, A., Taniuchi, A., May, T., Kawata, K. \& Okabe, S. Increased antibiotic resistance of Escherichia coli in mature biofilms. Appl. Environ. Microbiol. 75, 4093-4100 (2009).

44 Leriche, V., Sibille, P. \& Carpentier, B. Use of an enzyme linked lectinosorbent assay to monitor the shift in polysachharide composition in bacterial biofilms. Appl. Environ. Microbiol. 66, 1851-1856 (2000).

45 Yarwood, J. M. \& Schlievert, P. M. Quorum sensing in staphylococcal infections. J. Clin. Invest. 112, 1620-1625 (2003) 